\title{
HySafe SBEP-V20: Numerical studies of release experiments inside a naturally ventilated residential garage
}

\author{
E.A. Papanikolaou ${ }^{a, b, e, *}$, A.G. Venetsanos ${ }^{a}$, M. Heitsch ${ }^{b}$, D. Baraldi ${ }^{b}$, A. Huser ${ }^{c}$, J. Pujol ${ }^{c}$, \\ J. Garcia ${ }^{d}$, N. Markatos ${ }^{e}$ \\ ${ }^{a}$ Environmental Research Laboratory, National Centre for Scientific Research Demokritos, Aghia Paraskevi, Attikis 15310, Greece \\ ${ }^{b}$ European Commission DG-JRC, Institute for Energy - Cleaner Energy Unit, 1755 ZG Petten, The Netherlands \\ ${ }^{\circ}$ DNV Research and Innovation, Osio, Nonway \\ dEscuela Técnica Superior de Ingenieros Industriales, Universidad Politécnica de Madrid, José Gutiérrez Abascal, 2, E-28006 Madrid, Spain \\ e National Technical University of Athens, School of Chemical Engineering, Zografou 15780, Greece
}

\section{A R T ICLE IN F O}

Keywords:

Hydrogen safety

Garage

Release and dispersion simulations

Natural ventilation

CFD

Experiments

\begin{abstract}
A B S T R A C T
This work presents the results of the Standard Benchmark Exercise Problem (SBEP) V20 of Work Package 6 (WP6) of HySafe Network of Excellence (NoE), co-funded by the European Commission, in the frame of evaluating the quality and suitability of codes, models and user practices by comparative assessments of code results. The benchmark problem SBEPV20 covers release scenarios that were experimentally investigated in the past using helium as a substitute to hydrogen. The aim of the experimental investigations was to determine the ventilation requirements for parking hydrogen fuelled vehicles in residential garages. Helium was released under the vehicle for $2 \mathrm{~h}$ with $7.200 \mathrm{l} / \mathrm{h}$ flow rate. The leak rate corresponded to a $20 \%$ drop of the peak power of a $50 \mathrm{~kW}$ fuel cell vehicle. Three double vent garage door geometries are considered in this numerical investigation. In each case the vents are located at the top and bottom of the garage door. The vents vary only in height. In the first case, the height of the vents is $0.063 \mathrm{~m}$, in the second $0.241 \mathrm{~m}$ and in the third $0.495 \mathrm{~m}$. Four HySafe partners participated in this benchmark. The following CFD packages with the respective models were applied to simulate the experiments: ADREA-HF using $\mathrm{k}-\varepsilon$ model by partner NCSRD, FLACS using $\mathrm{k}-\varepsilon$ model by partner DNV, FLUENT using $\mathrm{k}-\varepsilon$ model by partner UPM and CFX using laminar and the low-Re number SST model by partner JRC. This study compares the results predicted by the partners to the experimental measurements at four sensor locations inside the garage with an attempt to assess and validate the performance of the different numerical approaches.
\end{abstract}

\section{Introduction}

A part of the Integrating Activities within the HySafe Network of Excellence (NoE) [1] was the collection of experiments in areas relevant to hydrogen safety for code and model benchmarking. The exercises proposed by the consortium partners were identified as SBEPs which stands for "Standard Benchmark Exercise Problems". Apart from validating the performance of the codes and models to reproduce the experimental data, a comparative assessment between them 
was aimed at identifying the main priority areas for further development of the codes and models and to provide recommendations for optimal tools and user best practices.

The benchmark problem SBEP-V20 is based on release scenarios that were experimentally investigated in the past by Swain et al. (1998) [2]. The latter work was a combined experimental and CFD research program in order to determine the ventilation requirements of residential garages to store hydrogen fuelled vehicles. Specifically, the work investigated the suitability of existing garages to store hydrogen fuelled vehicles and the need for any modifications. A fullscale model of a single car garage containing a vehicle was used. The experimental facility was located indoors to eliminate wind and outdoor temperature variations. During the course of the experiments and based on the results several modifications were investigated. Initially, the modifications covered natural ventilation which was provided by vents at the garage door. Additionally, a vent located at the ceiling was also investigated. Finally, forced ventilation and a hydrogen detection system were examined. Helium at a leak rate of $7.200 \mathrm{l} / \mathrm{h}$ located under the car was used as a surrogate to hydrogen for all experiments. The CFD calculations were performed using FLUENT. Calculations showed that the difference in hydrogen and helium concentrations in resembling geometries rarely exceeds $15 \%$. The largest differences occurred during the transient period before steady state and before the highest concentrations were reached. Papanikolaou et al. (2005) [3] presented the results of the simulations of three cases of the Swain garage experiments [2] using the standard $k-\varepsilon$ model. The results were generally in good agreement with the experiments.

Hydrogen releases in a hermetically sealed cylinder were presented by Gallego et al. (2007) [4]. The risks incurred by leaks in partially enclosed spaces, such as garages with natural ventilation, are affected by both the total volume of the released $\mathrm{H}_{2}$ and the flow rate at which $\mathrm{H}_{2}$ is being released [5]. Furthermore, the location of the vents strongly affects the risks. Vents located at the top of the enclosure allow $\mathrm{H}_{2}$ to exit provided that vents at the lower part of the enclosure are also present. The lower vents allow fresh air to enter and replace the $\mathrm{H}_{2}$ enriched mixture exiting from the upper vents. Swain et al. (1999) [6] performed both hydrogen dispersion experjments in simple vented enclosures and CFD calculations using the FLUENT code. The work showed the feasibility of using helium gas to verify CFD models which can then be used to predict the distribution and concentration of hydrogen gas in a leakage scenario. Agranat et al. (2004) [7] simulated the vented hallway experiment using the PHOENICS code and found results similar to the FLUENT code. In the paper of Swain and Shriber (1998) [8] a comparison of the safety risks of four types of vehicle fuels (hydrogen, natural gas, LPG and gasoline) release inside a single car garage was made using FLUENT code. It was found that only LPG and gasoline produced appreciable volumes of combustible gas. In another work, Breitung et al. (2001) [9] applied the GASFLOW CFD code to calculate the temporal and spatial distribution of hydrogen and applied criteria to evaluate the flame acceleration and detonation potential in an effort to estimate the combustion hazards, due to the boil-off from the cryogenic hydrogen tank of a car in a private garage. Parsons and Brinckerhoff (2004)
[10] made CFD calculations in order to evaluate the facility modifications and associated incremental costs that may be necessary to safely accommodate hydrogen fuel cell vehicles in four support facility case studies (commercial multi-story above-ground parking, commercial multi-story belowground parking, residential two vehicle garages and commercial maintenance/repair/service station). Paillere et al. (2005) [11] highlighted the importance of using CFD for safety assessment, design of mitigation systems and presented a validation of their in-house code in release, dispersion, combustion and mitigation scenarios. Barley et al. (2007) [12] provided relationships between design variables (vent area, height, discharge coefficient) for buoyancy-driven passive ventilation of $\mathrm{H}_{2}$ from a room. A simplified model was described and a CFD code was used to simulate a typical two-car garage with different $\mathrm{H}_{2}$ leakage rates scenarios. In a later work, Barley and Gawlik (2009) [13] presented experimental and CFD and algebraic modelling work of a lowvelocity helium leak inside a full-scale test room under nearly isothermal and steady conditions. The authors pointed out the significance of temperature difference between the inside and outside of the enclosure on the indoor hydrogen concentration since thermo-circulation can either oppose or augment the buoyancy-driven ventilation. Gupta et al. (2007) [14] provided a detailed experimental information on $\mathrm{H}_{2}$ dispersion inside a full-scale unventilated garage. The test cases evaluated the influence of injected $\mathrm{H}_{2}$ volumes and initial conditions on the dispersion and mixing characteristics inside the facility whereas He was used instead of $\mathrm{H}_{2}$. The authors concluded that the risk induced is most strongly affected by the total volume of the released gas rather than by the flow rate. Lowesmith et al. (2007) [15] presented an experimental work of gas (with varied $\mathrm{H}_{2} / \mathrm{CH}_{4}$ composition) release inside a ventilated enclosure. Analysis of data and predictions were done with the use of a simple mathematica] model. The authors concluded that both buoyancy and wind driven ventilation are important. The paper by Lacome et al. (2007) [16] presents test results of subsonic $\mathrm{H}_{2}$ releases in confined area. A comparison between $\mathrm{He}$ and $\mathrm{H}_{2}$ concentrations was also made. The authors stated that He can be used as a substitute to $\mathrm{H}_{2}$ although further experiments are necessary. The paper by Venetsanos et al. (2009) [17] presents a CFD inter-comparison of an experiment inside a garage with $1 \mathrm{~g} / \mathrm{s} \mathrm{H}_{2}$ vertical release. The simulations by 12 organizations using different codes and models before and after the experiment were evaluated. Large variation was found in the results during the pre-test phase whereas the variation was significantly reduced in the post-test phase. The paper by Papakonstantinou et al. (2003) [18] presented a simulation work of $\mathrm{CO}$ concentration inside a typical garage (with a capacity of 110 vehicles) with and without mechanical ventilation. The results showed that the numerical solutions were very effective for ventilation and design purposes. Duci et al. (2004) [19] presented simulations of $\mathrm{CO}$ inside a typical garage for three different ventilation rates and concluded that the CFD calculations can be obtained quickly and economically with considerable confidence.

This review shows not only the experimental work of $\mathrm{H}_{2}$ releases focused in enclosures (such as garages) but also the importance of using CFD codes as a tool for risk assessment, 
estimation of hazards and as a basis for suggestions to the design requirements of a garage to safely accommodate hydrogen vehicles. On the other hand the scenarios often include slow flow conditions (laminar or transitional), for which the choice of a model is not trivial. The selection of grid resolution and boundary conditions is another issue that needs to be addressed. Consequently, the development of CFD practice guidelines, based on extensive validation work for such scenarios is important.

This work focuses on the helium experiments by Swain et al. [2]. Three of these tests were selected as benchmark problem SBEP-V20. Four HySafe partners participated in this benchmark with different CFD packages and approaches. This study compares the results predicted by the partners to the experimental data at four sensor locations inside the garage with an attempt to assess and validate the performance of the different codes and models. Furthermore, the structure of the flow field and the effect of passive ventilation on the formation of flammable cloud are investigated.

As described by Barley and Gawlik (2009) [13], the temperature difference between the inside and the outside of the enclosure can effect significantly the ventilation and therefore the indoor hydrogen concentration. In the experiments by Swain et al. [2], the experimental facility was located indoors, creating isothermal conditions. Therefore the temperature difference issue was not dealt with, neither in the experiments nor in the simulations. It must be emphasized that in any CFD investigations of ventilation in real situations non isothermal conditions should be taken into account

\section{Experimental description}

The experimental facility represents a full-scale single car garage with dimensions $6.4 \times 3.7 \times 2.8 \mathrm{~m}$ and two vents on the door. Vent openings with varying height were examined. In al] cases the vents' width was the same as that of the door of the garage. A full-scale plywood model vehicle was placed inside the garage. All testing was done with helium as a surrogate to hydrogen. The helium flow rate was $7.200 \mathrm{l} / \mathrm{h}$ and the release lasted $2 \mathrm{~h}$. The leak location was at the bottom of the vehicle in the front part and centered at its width. The sensors were located at the four comers of the garage. Three of the Swain tests were selected as benchmark problem SBEP-V2O, Case 1 with $0.063 \mathrm{~m}$ vent height, Case 2 with $0.241 \mathrm{~m}$ vent height and Case 3 with $0.495 \mathrm{~m}$ vent height (Table 1). Fig. 1 shows the geometry and the dimensions of the experimental facility, the location of the leak, the vents and the four sensors. The geometrical details of the facility can be found in [2] and [3]. However, no information was available about the uncertainties of the measurements or the characteristics of the

Table 1 - Height of the two openings for each case.

\begin{tabular}{lc} 
Case & Height of the two openings (m) \\
\hline Case 1 - small openings & 0.063 \\
Case 2 - medium openings & 0.241 \\
Case 1 - large openings & 0.495 \\
\hline
\end{tabular}

sensors such as accuracy and detection limits. Some of the reported helium concentrations were very small especially at the lower sensors (ranging from 0 to $0.4 \%$ ), which usually involve relatively high measurement errors.

\section{Benchmark description}

Four HySafe partners participated in this benchmark with the following CFD packages and models: ADREA-HF [20] using $\mathrm{k}-\varepsilon$ model by partner NCSRD, FLACS [21] using $k-\varepsilon$ mode] by partner DNV, FLUENT [22] using $\mathrm{k}-\varepsilon$ mode] by partner UPM and CFX [23] using laminar and the low-Re number SST model by partner JRC. The main characteristics of the modelling approach of each participant are given in Tables 2 and 3 . The tables give the details of the modelling strategy that was adopted for the results that were submitted as "final results" for inter-comparison. Additionally, partners DNV, JRC and NCSRD performed grid convergence studies. DNV, JRC and NCSRD used also different models to examine whether they affect the results. DNV examined two different boundary conditions, the "symmetry" and "nozzle" and different time steps. Finally, NCSRD and JRC examined the effect of the computational domain size. The outcome of these studies will be discussed in the following section.

\section{Statistical performance indicators}

A useful method to evaluate the performance of different modelling approaches (predicted values) against a common experimental dataset (observed values) is statistical analysis. Four statistical performance indicators were used in this study. These indicators were recommended by Hanna (1989) [24] and Hanna et al. (1993) [25] for evaluating air dispersion models. However they are widely used in different studies. It is necessary to consider multiple performance indicators since each indicator has advantages and disadvantages and as there is not a single indicator that is universally applicable to all conditions.

Fractional Bias (FB): is the mean error that defines the residual of the observed $\left(C_{o}\right)$ and the predicted concentrations $\left(C_{p}\right)$. The bias is normalized to make it dimensionless. It has a value of 0 for an ideal model performance; it is non-linear and bounded by \pm 2 . Negative values indicate a model over prediction and positive values a model under prediction. The consequence of its non-linearity is that if a model tends to over or under predict considerably, though by quite different factors, the variance of the fractional bias will be low. A low variance in fractional bias can be taken as indicating confidence in the model predictions, but taken on its own this would be misleading. The expression of $\mathrm{FB}$ is given by:

$\mathrm{FB}=\frac{2\left(\overline{\mathrm{C}_{0}}-\overline{\mathrm{C}_{p}}\right)}{\overline{\mathrm{C}_{o}}+\overline{\mathrm{C}_{\mathrm{p}}}}$

where the overbar $(\bar{C})$ denotes the average over the dataset

Normalized Mean Square Error (NMSE): emphasizes the scatter of the entire dataset and is an estimator of the overall deviation between the observed and the predicted values. Smaller values of NMSE indicate better performance and are 


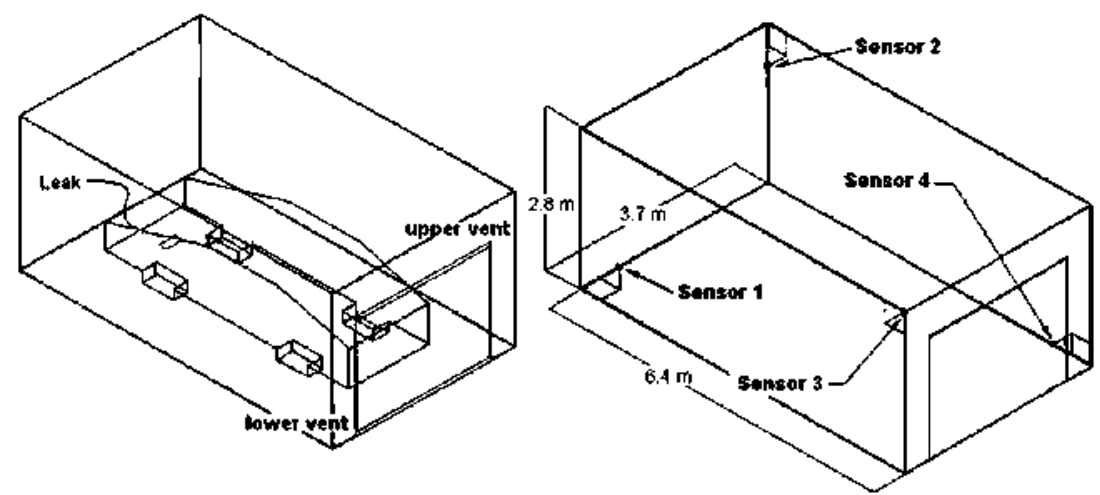

Fig. 1 - Geometry of the experimental facility (left-hand side) and location of sensors (right-hand side).

not biased towards models that over predict or under predict. It has a value of 0 for an ideal model performance. The expression of NMSE is given by:

NMSE $=\frac{\overline{\left(C_{o}-C_{p}\right)^{2}}}{\overline{C_{o}} \cdot \overline{C_{p}}}$

Geometric Mean Bias (MG): measures relative mean bias. Values of 0.5 and 2.0 can be thought of as 'factor of two' over and under predictions in the mean, respectively. It has a value of 1 for an ideal model performance. The expression of $M G$ is given by:

$M G=\exp \left[\left(\overline{\ln C_{o}}\right)-\left(\overline{\ln C_{p}}\right)\right]$

Geometric Mean Variance (VG): measures relative scatter. It has a value of 1 for an ideal model performance. A value of about 1.6 indicates a typical 'factor of two' scatter between individual pairs of observed and predicted values. The expression of VG is given by:

$V G=\exp \left[\left(\overline{\ln C_{o} / C_{p}}\right)^{2}\right]$

FB and MG are measures of mean bias and indicate only systematic errors, whereas NMSE and VG are measures of scatter and reflect both systematic and random errors.

Model acceptance criteria for the above statistical indicators based on extensive experience with evaluating models with many field experiments were made by Chang and Hanna
(2004) [26]. The following typical performance measures were suggested:

Mean bias within $\pm 30 \%$ of the mean (roughly $|\mathrm{FB}|<0.3$ or $0.7<\mathrm{MG}<1.3$ )

Random scatter about a factor of two to three of the mean (roughly NMSE $<1.5$ or VG $<4$ ).

\section{Results and discussion}

Several investigations on Case 1 were made by the partners. Partners DNV performed a time step independence study. Decreasing the time step from 0.01 to $0.001 \mathrm{~s}$ had no affect on the results. Grid sensitivity studies were performed by three partners, DNV, JRC and NCSRD. DNV reported that an increase of the control volumes by a factor of 8 resulted in small changes in the concentration at the sensors. JRC increased the number of grid nodes by a factor of 3.5, refining the mesh close to the vents and the leak, and found no effect. NCSRD decided to make a more uniform grid than the one used in their previous work [3]. The results were slightly jmproved at the lower sensors. Also, partner NCSRD assumed symmetry. NCSRD performed a simulation including the whole garage geometry to test the effect of the symmetry assumption and found no change in the results.

Table $\mathbf{2}$ - Main characteristics of the codes, models and discretization schemes used by the participants.
Participant
CFD code
Model
Discretization schemes

C: convective scheme, $\mathrm{T}$ : temporal scheme

\begin{tabular}{|c|c|c|c|}
\hline DNV & FLACS 9 & $\begin{array}{l}k-\varepsilon \text { standard } \\
\sigma_{\kappa}=1.0, \sigma_{\varepsilon}=1.3 \\
\sigma=0.7\end{array}$ & $\begin{array}{l}\text { SIMPLE for pressure-velocity coupling } \\
\text { C: 2nd order "kappa" scheme (blends upwind and central difference) } \\
\text { T: 1st order backward Euler }\end{array}$ \\
\hline JRC & CFX 11 SP 1 & Laminar, buoyant & $\begin{array}{l}\text { C: } 2 \text { nd order (high resolution) } \\
\text { T: } 2 \text { nd order backward Euler }\end{array}$ \\
\hline NCSRD & ADRA-HF & $\begin{array}{l}\mathrm{k}-\mathrm{k} \text { standard with buoyancy effects } \\
\sigma_{\mathrm{k}}=1.0, \sigma_{\varepsilon}=1.3 \\
\sigma=0.72\end{array}$ & $\begin{array}{l}\text { C: 1st order upwind } \\
\mathrm{T}: 1 \text { st order fully implicit }\end{array}$ \\
\hline UPM & FLUENT & $k-\varepsilon$ standard & $\begin{array}{l}\text { C: 2nd order upwind } \\
\text { T: 1st order implicit }\end{array}$ \\
\hline
\end{tabular}


Table 3 - Main characteristics of the mesh, time step and initial and boundary conditions used by the participants.

Participant Domain and grid characteristics Time step, CPU time, computer

Initial and boundary conditions

\begin{tabular}{|c|c|c|c|}
\hline DNV & $\begin{array}{l}\text { Extended domain by } 10 \% \text { garage length in } \\
x \text {-direction (Case } 1 \text { and } 2 \text { ), extended by } \\
55 \% \text { garage length in } x \text {-direction (Case } 3 \text { ) } \\
\text { Cartesian grid } \\
\text { Case } 1 \text { : } 13.000 \text { cells }(40 \times 13 \times 25), 0.1 \mathrm{~m} \text { in } \\
X, 0.2 \mathrm{~m} \text { in } Y, 0.06 \mathrm{~m} \text { in } \mathrm{Z} \text { minimum cell } \\
\text { size, } 0.31 \mathrm{~m} \text { in } X, 0.38 \mathrm{~m} \text { in } \mathrm{Y}, 0.18 \mathrm{~m} \text { in } \mathrm{Z} \\
\text { maximum cell size } \\
\text { Case } 2: 14.560 \text { cells }(40 \times 13 \times 28), 0.1 \mathrm{~m} \text { in } \\
X, 0.2 \mathrm{~m} \text { in } \mathrm{Y}, 0.04 \mathrm{~m} \text { in } \mathrm{Z} \text { minimum cell } \\
\text { size, } 0.31 \mathrm{~m} \text { in } \mathrm{X}, 0.38 \mathrm{~m} \text { in } \mathrm{Y}, 0.18 \mathrm{~m} \text { in } \mathrm{Z} \\
\text { maximum cell size } \\
\text { Case } 3: 16.575 \text { cells }(51 \times 13 \times 25), 0.1 \mathrm{~m} \text { in } \\
X, 0.2 \mathrm{~m} \text { in } \mathrm{Y}, 0.06 \mathrm{~m} \text { in } \mathrm{Z} \text { minimum cell } \\
\text { size, } 0.28 \mathrm{~m} \text { in } \mathrm{X}, 0.38 \mathrm{~m} \text { in } \mathrm{Y}, 0.18 \mathrm{~m} \text { in } \mathrm{Z} \\
\text { maximum cell size }\end{array}$ & $\begin{array}{l}\text { Time step: } 0.01 \mathrm{~s} \\
\text { System clock time: } 100,230 \text { and } 100 \mathrm{~h} \\
\text { for Case } 1,2 \text {, and } 3 \\
2 \mathrm{CPU} \text { DELL Intel Xeon } 2.66 \mathrm{GHz} \text { (code } \\
\text { runs with } 1 \mathrm{CPU} \text { ), } 2 \mathrm{~Gb} \text { RAM }\end{array}$ & $\begin{array}{l}\text { Temperature: } 20^{\circ} \mathrm{C} \text {, } \\
\text { Jet inlet: } 3.284 \cdot 10^{-4} \mathrm{~kg} / \mathrm{s}, 0.02 \mathrm{~m}^{2} \text { jet area, } \\
\text { "Nozzle" boundary condition to all } \\
\text { computational boundaries (ambient } \\
\text { pressure and zero-gradient to rest of } \\
\text { variables) } \\
\text { Ground and garage walls: No slip with log } \\
\text { law }\end{array}$ \\
\hline JRC & $\begin{array}{l}\text { Initial results: Extended domain by almost } \\
50 \% \text { and } 100 \% \text { in the } x \text { and } z \text {-directions } \\
418.945 \text { tetrahedral cells ( } 81.374 \text { nodes) } \\
\text { Final results: Extended domain by almost } \\
100 \% \text { in } x \text {-direction, } 100 \% \text { in } y \text { direction (in } \\
\text { both sides of the garage) and } 300 \% \text { in the } z- \\
\text { direction } \\
399.002 \text { tetrahedral cells ( } 77.082 \text { nodes) in } \\
\text { Case } 1 \text { to } 453.074 \text { tetrahedral cells ( } 88.819 \\
\text { nodes) in Case } 3\end{array}$ & $\begin{array}{l}\text { Time adaptive method with automatic } \\
\text { time step selection between } 0.1 \text { and } 1 \mathrm{~s} \\
\text { (average time step: } 0.25 \mathrm{~s} \text { ). } \\
\text { System clock time: } 106 \mathrm{~h} \text { (typical) } \\
\text { 8 Intel Xeon } 3 \mathrm{GHz}\end{array}$ & $\begin{array}{l}\text { Temperature: } 15^{\circ} \mathrm{C} \text {, } \\
\text { Jet inlet: } 3.4 \cdot 10^{-4} \mathrm{~kg} / \mathrm{s}, 0.1 \mathrm{~m} / \mathrm{s} \\
\text { Boundary conditions at open surfaces: } \\
\text { Sidewalls: symmetry plane } \\
\text { Top: opening (flow possible in all } \\
\text { directions) } \\
\text { Front: Outlet (flow only outwards) } \\
\text { Bottom and back side: Wall }\end{array}$ \\
\hline NCSRD & 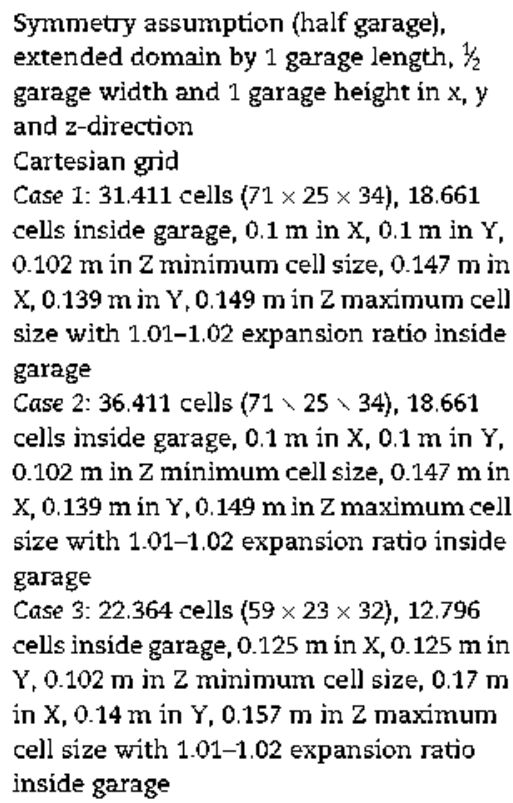 & $\begin{array}{l}\text { Maximum allowed time step: } 10^{-1} \mathrm{~s} \\
\text { System dock time: } 17,15 \text { and } 10 \mathrm{~h} \text { for } \\
\text { Case } 1-3 \\
\text { PC Windows, Intel Xeon CPU } 5160 \mathrm{~g} \\
3.00 \mathrm{GHz}, 4.00 \mathrm{~GB} \text { of RAM }\end{array}$ & $\begin{array}{l}\text { Temperature: } 20^{\circ} \mathrm{C} \text {, } \\
0.1 \mathrm{~m} / \mathrm{s}, 0.01 \mathrm{~m}^{2} \text { jet area } \\
\text { zero-gradient boundary conditions on } \\
\text { solid surfaces for He mass fraction, wall } \\
\text { functions for velocity, } \mathrm{k} \text { and } \mathrm{\varepsilon} \text {, symmetry } \\
\text { boundary conditions at symmetry plane, } \\
\text { inflow boundary conditions at the source, } \\
\text { no diffusion across source surface, at } \\
\text { other open surfaces of domain value for } \\
\text { normal velocities calculated from mass } \\
\text { balance (constant pressure), for other } \\
\text { variables zero-gradient if outflow or given } \\
\text { value (equal to the one at time } 0 \text { ) for inflow }\end{array}$ \\
\hline UPM & $\begin{array}{l}\text { Extended domain in the } x \text { and } z \text {-direction } \\
\text { by } 50 \% \\
320.272 \text { hexahedral cells. Resolution near } \\
\text { the source: } 0.044 \mathrm{~m} \text {. Maximum cell size: } \\
0.37 \mathrm{~m} \text {. }\end{array}$ & $\begin{array}{l}\text { Time step: } 0.1 \mathrm{~s} \text {. System clock time: } 80 \mathrm{~h} \\
4 \mathrm{CPU} \text { Intel Xeon } 2.66 \mathrm{GHz}\end{array}$ & $\begin{array}{l}\text { Temperature: } 20^{\circ} \mathrm{C} \text {, } \\
\text { Jet inlet: } 3.2 \cdot 10^{-4} \mathrm{~kg} / \mathrm{s}, 0.02 \mathrm{~m}^{2} \text { jet area, } \\
\text { Log law for garage walls, Boundary } \\
\text { conditions at open surfaces: fixed ambient } \\
\text { pressure }\left(1.013 \cdot 10^{5} \mathrm{~Pa}\right)\end{array}$ \\
\hline
\end{tabular}

Fig. 2 shows the predicted and experimental concentration series at the lower and upper sensors for Case 1 . The numerical results over predicted the experimental data. The mean experimental and simulated concentrations for each partner were used to quantitatively evaluate the performance of the modelling approaches and are shown in Fig. 4. The initial period of the release ( 0 to $1.000-2.000 \mathrm{~s}$ depending on the case) was not taken into account as the nearly steady-state concentration values were of interest. Fig. 4 shows that for Case 1 the over prediction for all partners is less than double for the upper sensors. Some partners predicted quite close to the experimental results for the lower sensors whereas the 

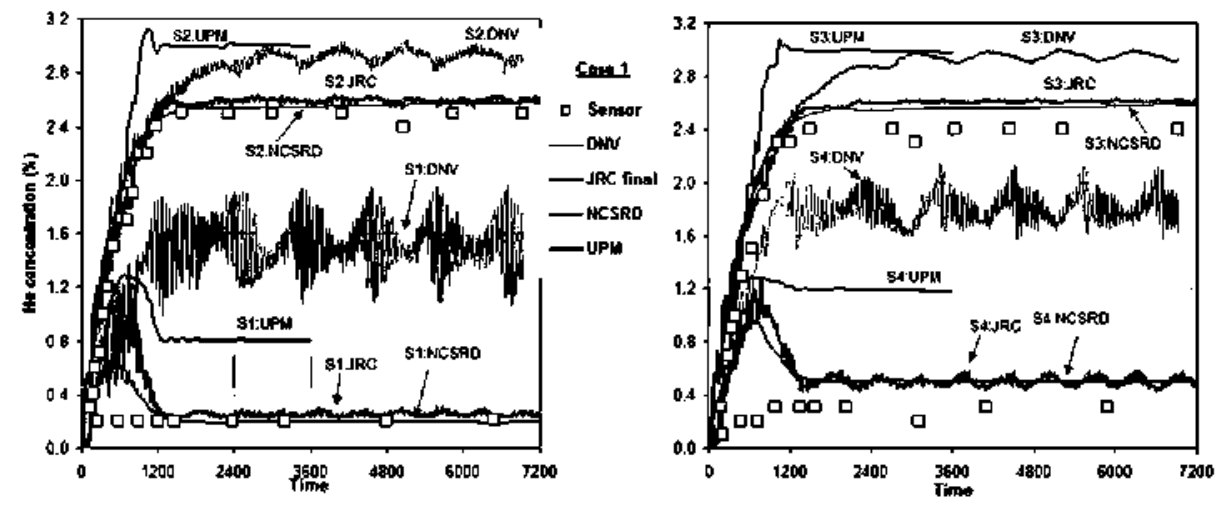

Fig. 2 - Case 1: Helium \% vol. concentration histories, left: sensors close to leak (S1, S2); right sensors close to openings (S3, S4).

rest over predicted higher than double. The results of partners NCSRD and JRC are in general the ones that were closer to the experimental for both upper and lower sensors.

The results from DNV show an oscillating pattern, especially for the lower sensors S1 and S4. Oscillations were also present in the results initially submitted by JRC. The oscillations were more apparent at the lower sensors for both DNV and JRC. Partners decided to investigate further this phenomenon. NCSRD decided to investigate the effect of the size of the computational volume on the results. One test was done subtracting the extra volume outside the garage in the $y$ direction while keeping the extra volume in the other two. Another test was done subtracting the extra volume in the $z$ direction but keeping the extra volume in the $x$ and $y$ directions. The results showed oscillations especially at the lower sensors for both tests. In all cases constant pressure boundary condition was used at the free boundaries. It should be noted that for the NCSRD results presented herein and in [3] the domain boundaries were far from the vent openings. In connection to the findings of NCSRD on the effect of the extra computational volume, JRC extended their domain in all 3 directions. Fig. 3 shows the initial and final results submitted by JRC at the lower sensors for Case 1. This figure shows not only the improvement of the final results in the concentration time series pattern but also in the mean value of the concentrations which were closer to the experimental. The computational domain used in the initial work by JRC was extended from the garage boundaries in the $x$ and $z$-directions by $50 \%$ and $100 \%$ respectively whereas in their final results the domain was extended in the $x$-direction by $100 \%$, in the $y$ direction by $100 \%$ (in both sides of the garage) and in $z$ direction by $300 \%$. Regarding the significance of the domain size DNV also reported slow convergence to steady state when the domain had a size equal to that of the garage. The domain used in the results submitted by DNV was extended only by $10 \%$ of the garage length in the $x$-direction.

Extending the computational domain outside the garage is a way to locate the boundary conditions at the free planes far enough to weakly influence, if at all, the flow inside the garage. It is believed that the different domain sizes selected by each partner caused the boundary conditions to influence the pressure distribution at the vents which in tum affected the flow inside the garage in a different manner for each partner. The sensitivity of the hydrogen concentration inside a naturally ventilated enclosure to the conditions at the vents was investigated recently by Matsuura et al. (2008) [27]. Hydrogen leaked inside a hallway at $9.44 \times 10^{-4} \mathrm{~m}^{3} / \mathrm{s}$ release rate which is comparable to the one of the current study. The computational domain had the same size as the hallway whereas constant pressure was imposed at the two vents. It was shown that the boundary conditions at the vents affected significantly the concentration distribution in the hallway since even a small decrease by $0.5 \mathrm{~Pa}$ of the pressure at the lower vent resulted in a substantial increase of the concentrations in the hallway, especially at the lower sensors.

Partners JRC and NCSRD investigated also the effect of the model applied. NCSRD assumed laminar flow and compared the results with the ones produced by the $k-\varepsilon$ model. The laminar model resulted in a minor increase of the concentrations at the lower sensors therefore the results were slightly worse. JRC applied the Shear Stress Transport (SST) turbulence model using their initial computational domain.

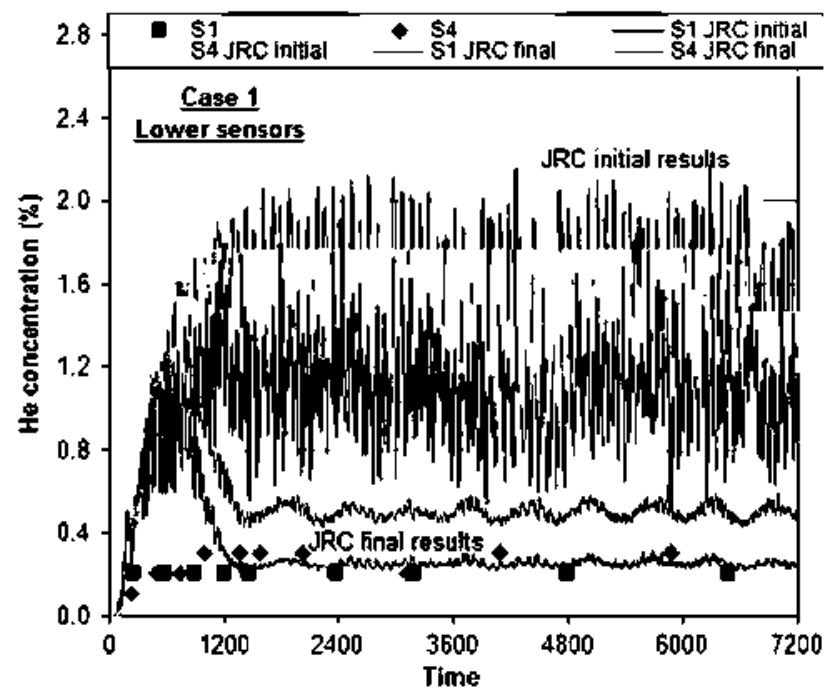

Fig. 3 - Initial and final results of Case 1 at lower sensors submitted by partner JRC. 


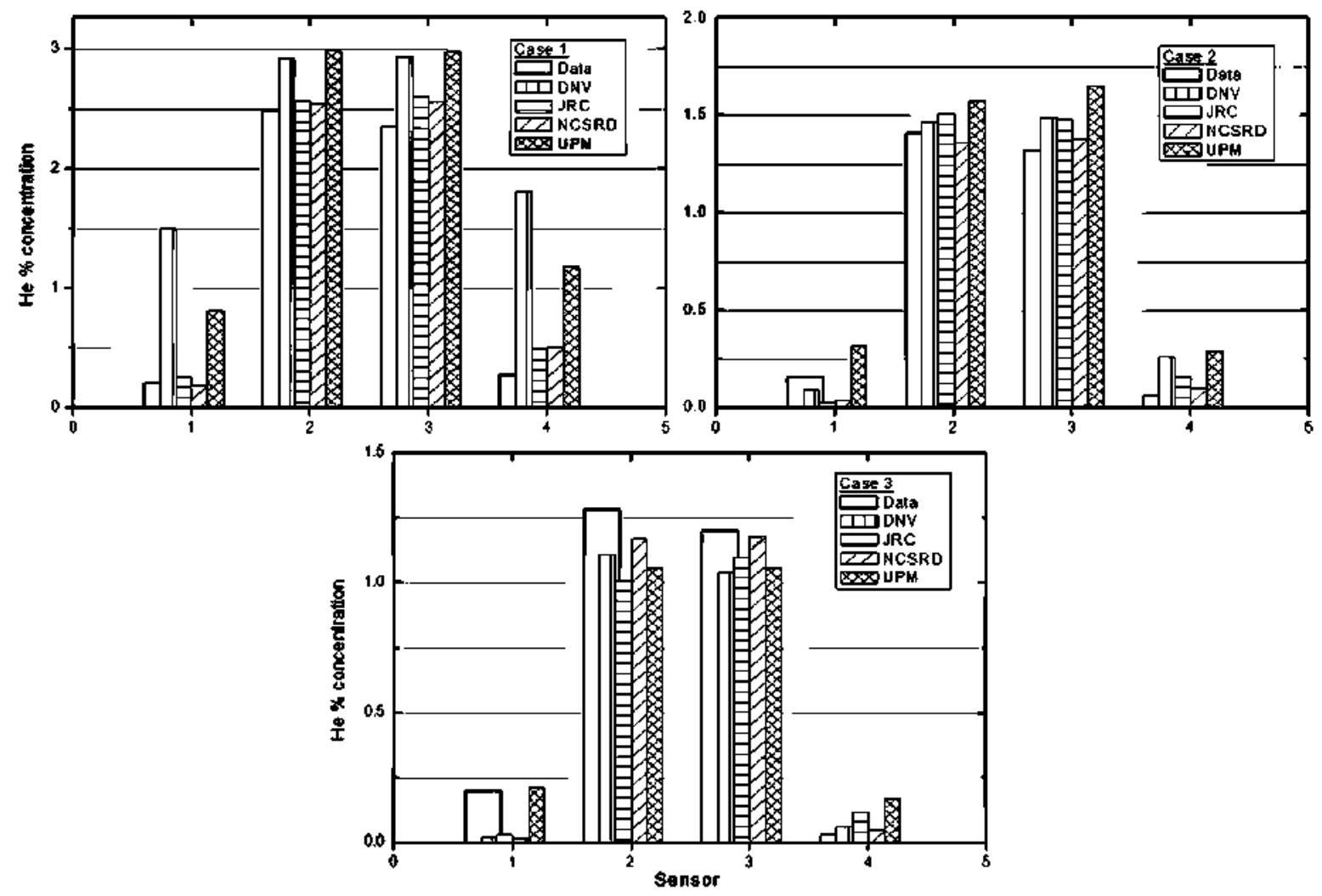

Fig. 4 - Mean experimental and simulated concentrations, upper left corner: Case; upper right comer: Case 2 and lower at the centre: Case 3.

The results were similar to the ones from the laminar model however the oscillations were slightly weaker.

The numerical results of the lower sensors of Case 1 show a peak before decreasing to steady state which is not shown in the experiment. This is due to a transient effect that reflects the time needed for the total mass flow to reach an established steady state inside the garage.

Fig. 5 shows the predicted and experimental concentration series at the lower and upper sensors for Case 2. The oscillations are now greatly reduced. DNV repeated the simulations for this case deactivating the turbulence model with the same computational domain. Their predictions were slightly more unstable but this effect was not significant. As it can be seen also from Fig. 4, there is again a general tendency to over predict the experimental data. Also, the results of the partners with the larger computational volume outside the garage were generally closer to the experimental.

The results by partner UPM, especially the ones for the lower sensors, show a significant rise of the concentrations which decrease when steady state is achieved indicating a stronger transient effect. Partner UPM attributed this behaviour to the coarse time step selected $(0.1 \mathrm{~s})$ in combination with the time discretization scheme (1st order) used.

Fig. 6 shows the predicted and experimental concentration series at the lower and upper sensors for Case 3 . The partners either over predicted or under predicted the concentration at the sensor close to the leak. Most of the partners under predicted the concentrations at the upper sensors whereas for the sensor close to the vent, all partners over predicted the experimental data (also shown in Fig. 4). Slight oscillations appear in the DNV results. DNV performed time step independence study by reducing the time step to $0.001 \mathrm{~s}$. The reduction did not affect the results. JRC performed a calculation with a low-Re number extension of the SST turbulence model using the initial computational domain. The mesh inside the garage was modified to resolve the boundary layer at the walls and the vehicle. The predictions for the two upper sensors in the garage were compared to the laminar calculation but did not show different time histories. Finally, DNV reported an unrealistic recirculation of helium from the upper vent to the lower which disappeared by extending the domain by $55 \%$ of garage length in the $x$-direction and by using "nozzle" boundary conditions at the free domain planes.

Generally, the results were worse for the lower sensors than the upper sensors. An explanation of the differences between the partners' results and a comparison with the experimental data was provided above. However, experimental uncertainties especially at the lower sensors where the concentrations were very low should also be borne in mind. The accuracy of the measurements and the detection limits of the sensors were not reported by experimentalists whereas some of the experimental results were not completely stable. The repeatability of experimental results was also not reported. Lastly, the environmental conditions were not given. Partners assumed different temperature as initial condition (see Table 3) which resulted in differences in 

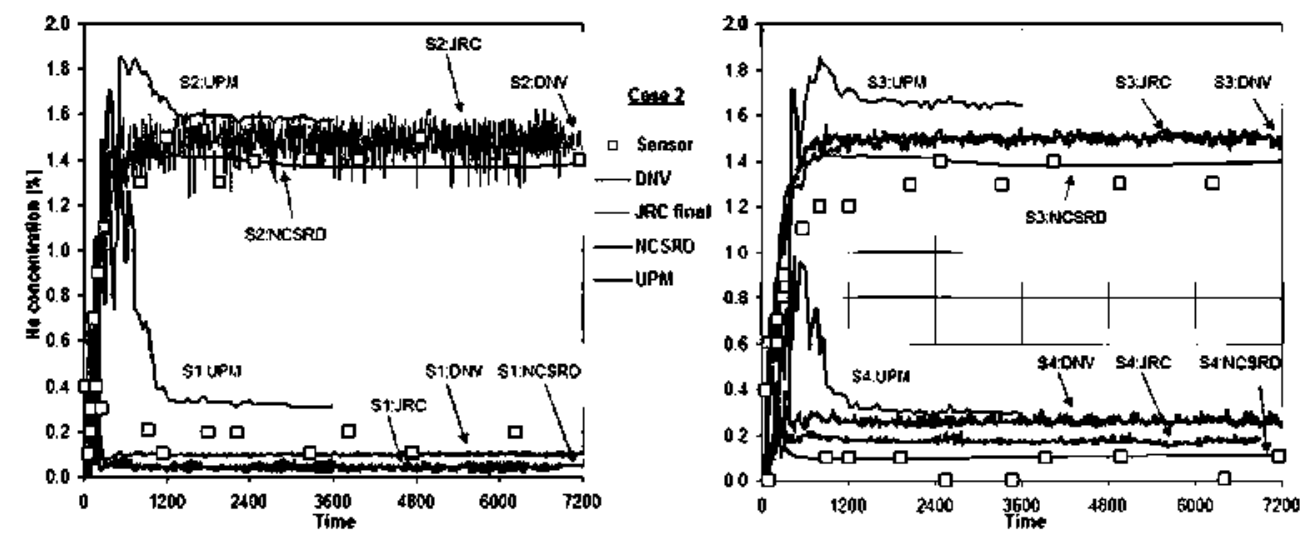

Fig. 5 - Case 2: Helium \% vol, concentration histories, left: sensors close to leak (S1, S2); right: sensors close to openings (S3, S4).

the release mass flow rate. However, the differences did not exceed $4 \%$ between partners therefore this parameter is not considered to affect the results significantly. To draw a safer conclusion on the comparison between the experimental data and the numerical results, additional information on the experimental conditions and apparatus used would be necessary.

Table 4 shows the values of the statistical performance indicators of the numerical simulation of each partner separately and of the overall results of all partners. The table also shows the ideal values for a perfect match between a model and the experimental data and the acceptance criteria of the indicators as mentioned in the previous paragraph. The shaded blocks indicate performance that falls outside these acceptance criteria.

Table 4 shows that the MG values are the ones that fall outside the acceptance criteria the most; followed by VG values and lastly FB. MG and VG values are known to be strongly influenced by extremely low observed and predicted values and are undefined for zero values. Chang and Hanna (2004) [26] suggested that when calculating MG and VG it would be useful to impose a minimum threshold for data values. It was also recommended that an instrument threshold such as the limit of detection be used as the lower bound for both experimental/observed and predicted values used to calculate the statistical performance indicators. Unfortunately this suggestion could not be realized in this work, since the detection limit of the sensors was not reported. The table shows that for Case 1 the errors in the predictions are more systematic whereas in Case 3 the errors are both systematic and random. It seems that as the vent height increases from Case 1 to Case 3 , the nature of the errors shifts from systematic to systematic and random.

As mentioned above, for a modelling approach to perfectly match the experimental data, the values of MG and VG should be equal to 1 . The following graphs show the values of MG versus VG for each partner and each case. The parabola represents the minimum possible value of VG corresponding to a particular MG due to systematic bias. This means that all points must lie within or above the parabola. Numerical results that produce points close to the parabola indicate a systematic error without random scatter whilst the ones that are closer to the central axis signify scatter. Fig. 7 shows that all partners over predicted the experimental data for Case 1 with JRC and NCSRD being the partners with the closest prediction.

Fig. 8 is the plot of the geometric variance (VG) versus the geometric mean (MG) for Case 2. Most of the partners are
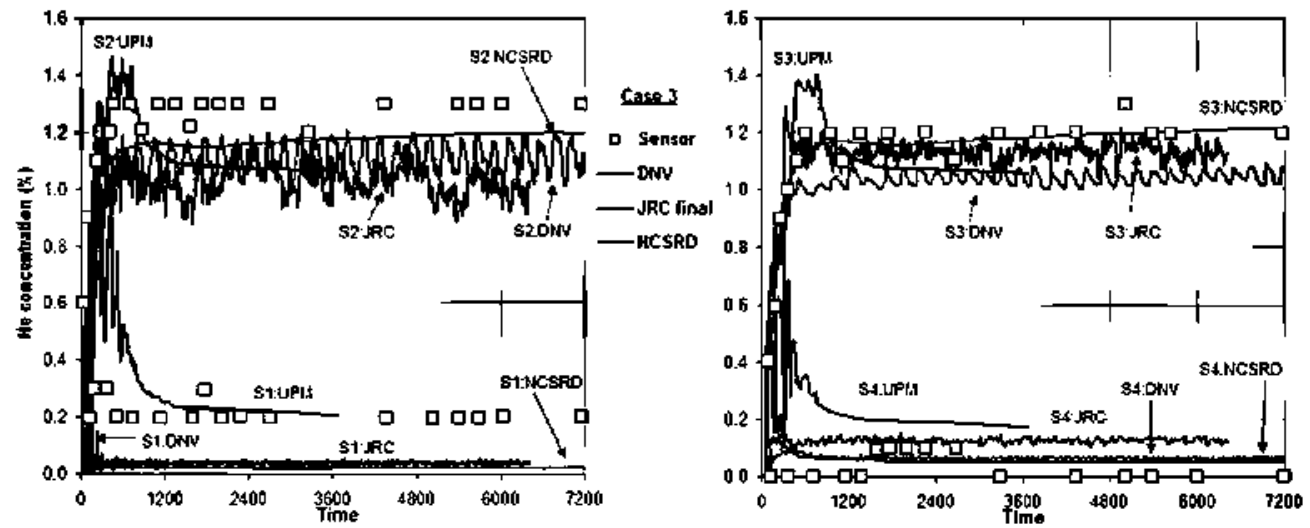

Fig. 6 - Case 3: Helium \% vol. concentration histories, left: sensors close to leak (S1, S2); right: sensors close to openings (S3, S4). 
Table 4 - Statistical parameters for each partner and overall performance of numerical results.

\begin{tabular}{|c|c|c|c|c|}
\hline \multirow[t]{2}{*}{$\begin{array}{l}\text { Statistical } \\
\text { parameters }\end{array}$} & \multicolumn{2}{|c|}{ Systematic errors } & \multicolumn{2}{|c|}{$\begin{array}{c}\text { Systematic and } \\
\text { unsystematic errors }\end{array}$} \\
\hline & FB & MG & NMSE & VG \\
\hline ldeal value & 0 & 1 & 0 & 1 \\
\hline $\begin{array}{c}\text { Acceptance } \\
\text { criteria }\end{array}$ & $|F B|<0.3$ & $0.7<\mathrm{MG}<1.3$ & $<1.5$ & $<4$ \\
\hline \multicolumn{5}{|l|}{ Case 1} \\
\hline DNV & -0.53 & 0.35 & 0.37 & \\
\hline $\mathrm{JRC}$ & -0.11 & 0.79 & 0.01 & 1.10 \\
\hline NCSRD & -0.09 & 0.86 & 0.01 & 1.09 \\
\hline UPM & -0.39 & 0.45 & 0.17 & 2.71 \\
\hline Overall & -0.33 & 0.54 & & 2.34 \\
\hline \multicolumn{5}{|l|}{ Case 2} \\
\hline DNV & -0.12 & 0.77 & 0.03 & 1.87 \\
\hline $\mathrm{JRC}$ & -0.07 & 1.13 & 0.03 & 2.57 \\
\hline NCSRD & 0.02 & 1.24 & 0.01 & 1.73 \\
\hline UPM & -0.26 & 0.52 & 0.08 & 2.13 \\
\hline Overall & -0.15 & 0.77 & & 2.15 \\
\hline \multicolumn{5}{|l|}{ Case 3} \\
\hline DNV & 0.19 & 1.6 & 0.06 & \\
\hline $\mathrm{JRC}$ & 0.18 & 1.23 & 0.08 & \\
\hline NCSRD & 0.12 & 1.69 & 0.02 & \\
\hline UPM & 0.08 & 0.69 & 0.05 & 2.15 \\
\hline Overall & 0.06 & 1.01 & & 3.91 \\
\hline
\end{tabular}

within the dotted vertical lines however there is no clear tendency to over predict or under predict the experimental data. Partners with a computational domain extended to all directions outside the garage under predicted the data whereas the rest over predicted. Furthermore, the results have now moved away from the parabola and lie closer to the central axis. This means that the nature of the errors is not only systematic, as in the previous case.

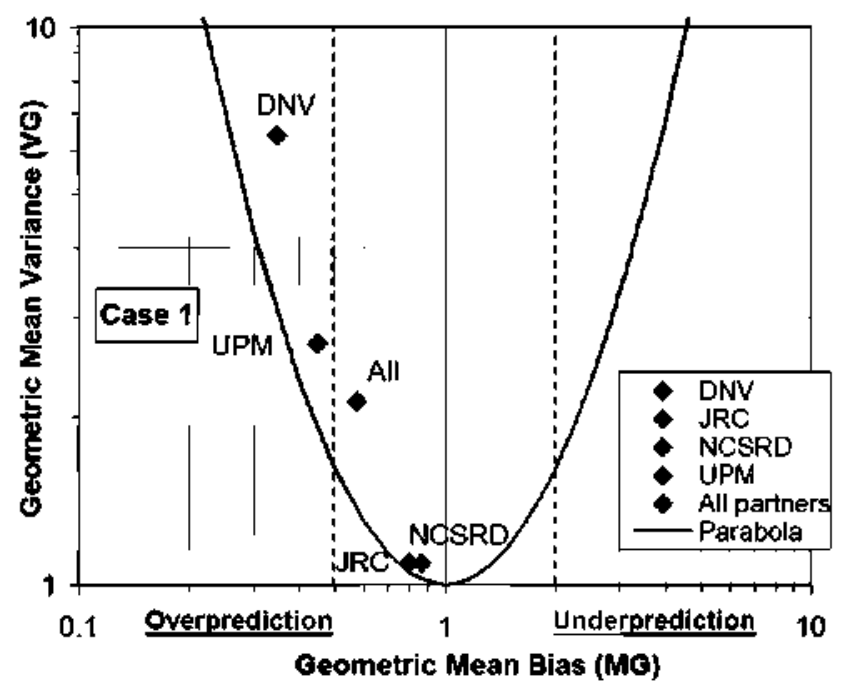

Fig. 7 - Plot of geometric mean (MG) versus geometric variance (VG) for Case 1. The vertical dotted lines at MG $=0.5$ and 2.0 represent a factor of two over prediction and under prediction. The parabola indicates minimum VG for a value of MG due to systematic bias.

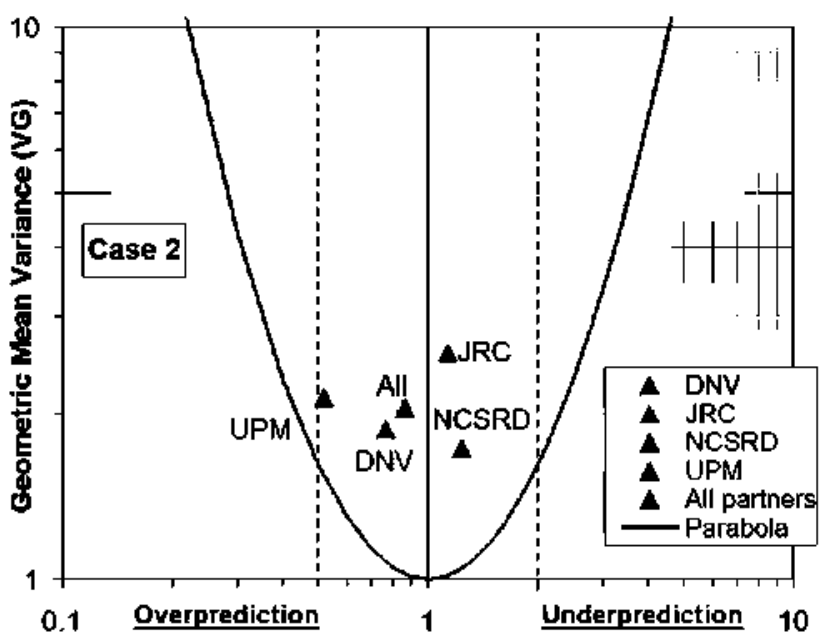

Ceometric Mean Blas (MG)

Fig. 8 - Plot of geometric mean (MG) versus geometric variance (VG) for Case 2.

Fig. 9 is the plot of the geometric variance (VG) versus the geometric mean (MG) for the case with the wider vent openings, Case 3 . In this case most of the results lie on the under prediction area of the plot and as in the previous case, the nature of the errors are both systematic and random.

One parameter for assessing the risk of an accidental release is the flammable mixture volume which is the volume of the air-hydrogen mixture, where hydrogen concentration is within the lower and upper flammability limits (4-75\%). Fig. 10 shows the predicted flammable mixture volume results submitted by partners JRC, NCSRD and UPM and also the helium mass contained in this cloud submitted by partners JRC and NCSRD time series for Case 1. The higher predictions for the flammable volume were given by UPM, followed by JRC whereas the lowest were reported by NCSRD. For this case, the value of the flammable volume and mass was $0.12 \mathrm{~m}^{3} / 1.6 \mathrm{~g}$,

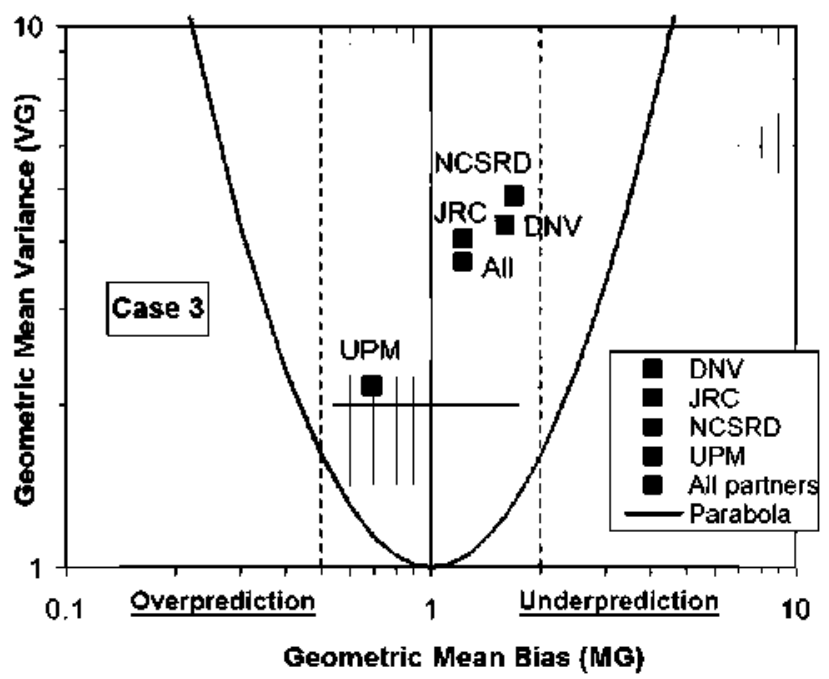

Fig. 9 - Plot of geometric mean (MG) versus geometric variance (VG) for Case 3. 


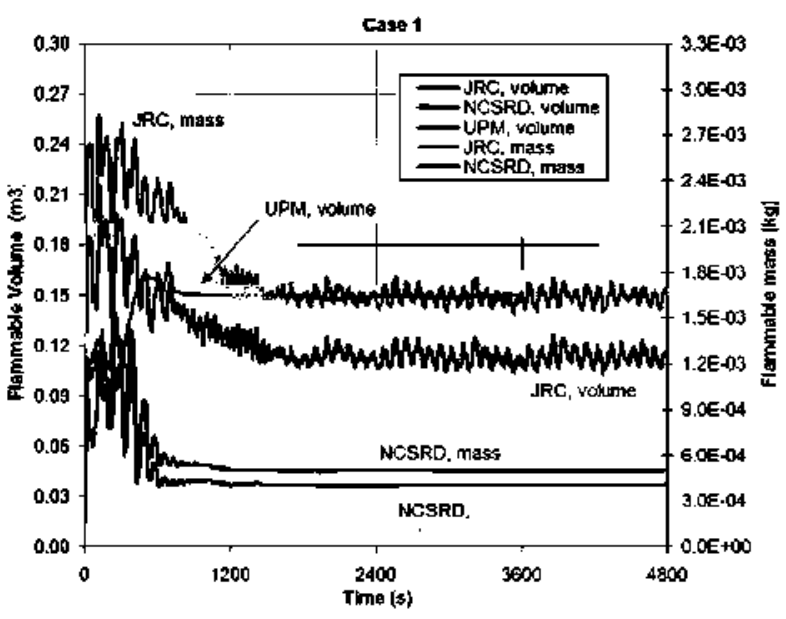

Fig. 10-Predicted flammable mixture volume and $\mathbf{H 2}$ mass histories for Case 1.

$0.15 \mathrm{~m}^{3}$ and $0.04 \mathrm{~m}^{3} / 0.5 \mathrm{~g}$ for JRC, UPM and NCSRD respectively. The values of the flammable volume and mass of JRC are roughly double than the ones reported by NCSRD. However, these two partners had very similar concentration results and very close to the experimental data in the four sensor locations inside the garage. Clearly, more experimental information such as concentration readings in several locations inside the garage and some general information on the size of the gas cloud (such as Background-Oriented Schlieren or other technique for flow visualization) are needed in order to validate the CFD models for the relevant parameters. The predicted flammable volume and mass of the same partners for Case 2 and 3 had the same tendency as in Case 1 thus the graphs was not included in this paper.

\section{Conclusions}

A comparison between the experimental and numerical results of the SBEP-V20 of WP6 of HySafe was presented. Four HySafe partners participated in this benchmark with 4 different codes (ADREA-HF, CFX, FLACS and FLUENT) using different models $(k-\varepsilon$, laminar, SST, low-Re number SST). This study compared the results predicted by the partners to the experimental measurements with an attempt to assess and validate the performance of the codes and models.

General agreement between the partners' predictions and the experimental data was good with tendency to overestimate the results of the upper sensors for the small and medium vent sizes and under estimate for the large vent size. The results of the lower sensors were generally over predicted for the small and medium vent sizes whereas for the larger vent size the partners either over predicted or under predicted at the sensor close to the leak and over predicted at the sensor close to the vent.

Laminar model over estimates slightly more than $\mathrm{k}-\varepsilon$ model the experimental data, as expected due to the lower diffusion. However, the different models used by the partners in general (laminar, k-e and SST) did not influence the results strongly, thus more experimental data and a more systematic comparison of the models is needed in order to suggest which one is more suitable than the rest for such scenarios.

The comparison of the simulations showed that the boundary conditions directly affect the flow in and out of the garage and contribute significantly to oscillating concentration results at the sensors as reported by some partners. Not only the selection of the boundary conditions must be done with care but also the distance of the boundary planes to the garage itself should be sufficient enough in all directions in order to influence as weak as possible the flow inside the garage since partners who placed the boundary planes far enough from the garage, produced results where the oscillations were either significantly reduced or completely diminished. Furthermore, it was found that when the boundary conditions were far from the garage the concentration in the four sensor locations was reduced, improving the accuracy of the simulation predictions.

Sufficient mesh resolution close to the vents and the source is recommended.

The statistical performance evaluation of the different CFD approaches showed that for the case with the smallest openings the errors in the predictions were more systematic, whereas for the case with larger openings the errors were both systematic and random. The nature of the results for the case with the medium openings falls in between the two other cases. The geometric mean followed by the geometric variance of the CFD predictions performed the worst. These parameters are known to be strongly influenced by extremely low values. Unfortunately, the guidelines by Chang and Hanna (2004) [26] to impose a minimum threshold for data and numerical results could not be realized lacking technical information on the sensors used in the experiments.

Partners, with similar and close to the experiments helium concentration results, predicted different flammable mixture volume and mass. These parameters were not provided as experimental data whereas the number of sensors was so limited that it was not possible to extract relevant information about the flammable cloud from them. Elaborated experimental information is needed in order to validate the different CFD approaches for these parameters.

Finally, the experiments were done indoors, thus phenomena that could affect the ventilation such as wind and temperature difference between indoors and outdoors were absent. Future validation of CFD modelling on natural ventilation scenarios should address the weather conditions that are likely to be found in real situations.

\section{Acknowledgments}

The authors would like to thank the European Commission for co-funding of this work in the framework of the HySafe FP6 Network of Excellence (contract no. SES6-CT-2004-502630).

\section{REFERENCES}

[1] HySAFE. Safety of hydrogen as an energy carrier. Network of Excellence, FP6, Available from, www.hysafe.org; 2003-2009. 
(2) Swain MR, Grilliot ES, Swain MN. Phase 2; risks in indoor vehicle storage, in Addendum to Hydrogen Vehicle Safety Report: Residential Garage Safety Assessment, analysis conducted by: Michael R. Swain, University of Miami, under subcontract to Directed Technologies Inc, prepared for the Ford motor company under prime Contract No. DE-AC0294 CE50389 to the U.S. Department of Energy, Office of Transportation Technologies; 1998.

[3] Papanikolaou EA, Venetsanos AG. CFD modelling for helium releases in a private garage without forced ventilation. In Proceedings of the 1st International Conference on Hydrogen Safety, Pisa; 2005.

[4] Gallego E, Migoya E, Martin-Valdepenas JM, Crespo A, Garcia J, Venetsanos AG, et al. An inter-comparison exercise on the capabilities of CFD models to predict distribution and mixing of $\mathrm{H}_{2}$ in a closed vessel. Intemational Journal of Hydrogen Energy 2007;32(13):2235-45.

[5] Swain MR, Filoso P, Grilliot ES, Swain MN. Hydrogen leakage into simple geometric enclosures. International Journal of Hydrogen Energy 1999;28:229-48.

[6] Swain MR, Grilliot ES, Swain MN. Experimental verification of a hydrogen risk assessment method. Chemical Health and Safety; 1999:28-32.

[7] Agranat V, Cheng Z, Tchouvelev A. CFD modelling of hydrogen releases and dispersion in hydrogen energy station. In Proceedings of the WHEC-15, Yokohama; 2004

[8] Swain MR, Shriber J. Comparison of hydrogen, natural gas, liquefied petroleum gas and gasoline leakage in a residential garage. Energy and Fuels 1998;12:83-9.

[9] Breitung W, Necker G, Kaup B, Veser A. Numerical simulation of hydrogen in a private garage. In Proceedings of the 4 th international symposium on hydrogen power-theoretical and engineering solutions - Hypothesis IV, Stralsund, Germany; 2001.

[10] Support facilities for hydrogen fuelled vehicles-Conceptual design and cost analysis study, Technical report. Prepared for California Fuel Cell Partnership by Parsons and Brinckerhoff in association with TIAX and University of Miami; 2004.

[11] Paillere $\mathrm{H}$, Studer E, Beccantini A, Kudriakov S, Dabbene F, Perret $\mathrm{C}$. Modelling of $\mathrm{H}_{2}$ dispersion and combustion phenomena using CFD codes. In Proceedings of the 1st International Conference on Hydrogen Safety, Pisa; 2005.

[12] Barley CD, Gawlik K, Ohi J, Hewett R. Analysis of buoyancydriven ventilation of hydrogen from buildings. In Proceedings of the 2nd International Conference on Hydrogen Safety, San Sebastian, Spain; 2007.

[13] Barley CD, Gawlik K. Buoyancy-driven ventilation of hydrogen from buildings: laboratory test and model validation. International Journal of Hydrogen Energy 2009;32: 5592-603.

[14] Gupta S, Brinster J, Studer E, Tkatschenko I. Hydrogen related risks within a private garage: concentration measurements in a realistic full scale experimental facility. In Proceedings of the 2nd International Conference on Hydrogen Safety, San Sebastian, Spain; 2007.

[15] Lowesmith BJ, Hankinson G, Spataru C, Stobbart M. Gas build-up in a domestic property following releases of methane/hydrogen mixtures. Intemational Journal of Hydrogen Energy 2009;34(14):5932-9.

[16] Lacome JM, Dagba Y, Jamois D, Perrette L, Proust Ch. Largescale hydrogen release in an isothermal confined area. In Proceedings of the 2nd International Conference on Hydrogen Safety, San Sebastian, Spain; 2007.

[17] Venetsanos AG, Papanikolaou E, Delichatsios M, Garcia J, Hansen $O R$, Heitsch $M$, et al. An inter-comparison exercise on the capabilities of CFD models to predict the short and long term distribution and mixing of hydrogen in a garage. International Journal of Hydrogen Energy 2009;34(14):591223.

[18] Papakonstantinou K, Chaloulakou A, Duci A, Vlachakis N, Markatos NC. Air quality in an underground garage: computational and experimental investigation of ventilation effectiveness. Energy and Buildings 2003;35(9):933-40.

[19] Duci A, Papakonstantínou K, Chaloulakou A, Markatos NC. Numerical approach of carbon monoxide concentration dispersion in an enclosed garage. Building and Environment 2004;39(9):1043-8.

[20] ADREA-HF code, http//www2.ipta.demokritos.gr/pages/ ADREA-HF.html.

[21] FLACS code, http:/www gexcon.com.

[22] FLUENT code, http//fluent.com/software/fluent/.

[23] ANSYS Inc.. Southpointe, 275 Technology Drive Canonsburg, USA, http:/www.ansys.com/products/cfx.asp.

[24] Hanna SR. Confidence limits for air quality model evaluations as estimated by bootstrap and jackknife resampling methods. Atmospheric Environment 1989;23: 1385-98.

[25] Hanna SR, Chang JC, Strimaitis DG. Hazardous gas model evaluation with field observations. Atmospheric Environment 1993;27A:2265-85.

[26] ChangJC, Hanna SR. Air qualitymodel performance evaluation. Meteorology and Atmospheric Physics 2004;87:167-96.

[27] Matsuura K, Kanayama $H$, Tsukikawa $H$, Inoue $M$. Numerical simulation of leaking hydrogen dispersion behaviour in a partially open space. International Journal of Hydrogen Energy 2008;33:240-7. 